\title{
PET Microplastics Affect Human Gut Microbiota Communities During Simulated Gastrointestinal Digestion. First Evidence of Plausible Polymer Biodegradation During Human Digestion
}

\author{
Alba Tamargo \\ CIAL, (CSIC-UAM) \\ Natalia Molinero \\ CIAL, (CSIC-UAM) \\ Julián J. Reinosa \\ Encapsulae S.L, Castellón de la Plana \\ Victor Alcolea-Rodríguez \\ Instituto de Catálisis y Petroleoquímica \\ Raquel Portela \\ Instituto de Catálisis y Petroleoquímica \\ Miguel A. Bañares \\ Instituto de Catálisis y Petroleoquímica \\ Jose F. Femández \\ Instituto de Cerámica y Vidrio, CSIC. c/ Kelsen \\ M.Victoria Moreno-Arribas ( $\square$ victoria.moreno@csic.es) \\ CIAL, (CSIC-UAM)
}

\section{Research Article}

Keywords: food-derived microplastics, PET, oral uptake, gastrointestinal digestion, intestinal microbiota, human health, simgi $\AA^{\circledR}$, MPs monitoring and transformation

Posted Date: June 15th, 2021

DOI: https://doi.org/10.21203/rs.3.rs-600797/v1

License: (c) (i) This work is licensed under a Creative Commons Attribution 4.0 International License. Read Full License

Version of Record: A version of this preprint was published at Scientific Reports on January 11th, 2022. See the published version at https://doi.org/10.1038/s41598-021-04489-w. 


\section{Abstract}

Microplastics are a widely recognized global problem due to their prevalence in natural environments and the food chain. However, the impacts of microplastics on human microbiota and their possible biotransformations during the gastrointestinal tract have not been well reported. To evaluate the potential risks of microplastics at the digestive level, completely passing a single dose of polyethylene terephthalate (PET) through the gastrointestinal tract was simulated by combining a harmonised static model and the dynamic gastrointestinal simgi® model, which recreates the different regions of the digestive tract in physiological conditions. PET MPs started several biotransformations in the gastrointestinal tract and appeared to expose the colon in ways that were structurally different from the original forms. We report that the feeding with microplastics alters human microbial colonic community composition, and hypothesize that some members of the colonic microbiota could adhere to MPs surface promoting the formation of biofilms. The work presented here indicates that microplastics are indeed capable of digestive-level health effects. Considering this evidence and the increasing exposure to microplastics in consumer foods and beverages, the impact of plastics on the functionality of the gut microbiome and their potential biodegradation through digestion and intestinal bacteria merits critical investigation.

\section{Introduction}

The prevalence of plastic particles in the food chain ${ }^{1-4}$ has raised the concern about health effects of microplastics ingestion. However, the risk assessment of microplastics' intake is still a global challenge for the scientific community ${ }^{2,5-7}$. Microplastics (MPs) are plastic particles with micrometer size that can be intentionally manufactured (primary MPs) or released to the environment from larger plastics (secondary MPs). The latter are able to act as pathogen microorganism's vectors ${ }^{8}$, and are referred to as a "cocktail of contaminants" due to their association with heavy metals, plastic additives or other persistent organic pollutants ${ }^{3}$. The most common polymers in Europe are, in decreasing order of production, polyethylene (PE), polypropylene (PP), polyvinyl chloride (PVC), polyurethane (PUR), and polyethylene terephthalate (PET) ${ }^{9}$. Among them, PET is the main polymer detected from plastic bottles used for beverages such as soft drinks, energy drinks, cold tea, and beer ${ }^{10}$ which is probably due to the bottle degradation, although MP particles have been also detected in mineral waters from glass bottles ${ }^{7}$.

PET is made from petroleum-derived terephthalic acid and ethylene glycol produced by melt-phase condensation and further solid-state polymerization. The presence of PET particles in human faeces observed in a pilot prospective study suggests their active interaction with the human digestive system ${ }^{11}$. However, their changes during gastrointestinal digestion or colonic fermentation are scarcely explored. To date, only Stock et al. have simulated in vitro human digestion of several MP particles along the gastrointestinal tract ${ }^{12}$. They did not observe pronounced structural changes due to digestion fluids except for the formation of a surface organic corona around the MP particles. However, this recent study did not explore the colonic stage or MP interactions with colonic microbiota ${ }^{12}$. The primary health effects of food-ingested plastic particles are triggered from the digestive system, causing direct damage not only at local level, as irritation or intestinal dysbiosis, but also at systemic level ${ }^{4}$. In this regard, Fackelmann and Sommer reviewed the link between MP-induced gut dysbiosis and host health and suggested that MPs could impact gut microbiome and that chronic exposition could infer gut dysbiosis in several species ${ }^{13}$. Still, the effect of microplastics on the human gut microbiome is uncertain. Few studies have investigated this issue, just in animal models, and with much higher MPs doses than those detected in edible foods and beverages 14-16. Their most common observation has been that exposure to MPs altered gut bacterial diversity and caused other health negative effects including changes in gut metabolic profiles and inflammation.

The human digestion of MPs and their effects on colonic microbiota can be studied with different approaches, including in vitro and in vivo approaches. Due to their physiological relevance, investigations with animals and human trials generally offer the most precise results and are still considered the 'gold standard' for certain diet-related questions. However, due to ethical restrictions, high cost, and the complexity of the multistage processes of human digestion, there is a real need for in vitro models that mimic the physiological conditions of human digestion. Static models can be used to determine endpoints of digestion or kinetics of very specific steps of digestion, such as stomach and small intestinal biotransformations. In contrast, dynamic models are more complex, but closer to the physiological reality of the gastrointestinal tract ${ }^{17,18}$. One of these dynamic simulators is the simgi® system, a computer-controlled gastrointestinal in vitro model designed to reproduce the colonic microbiota responsible for metabolic bioconversions in the large intestine that has been employed to study the effect of different foods ${ }^{19-25}$. In addition, dynamic simulators like simgi® have been shown to be also useful to evaluate the effects on intestinal microbiota of Active Pharmaceutical Ingredients (API) ${ }^{26}$, heavy metals ${ }^{27,28}$ and food-use nanomaterials ${ }^{29,30}$.

In this work, PET MPs in amounts that were in the range of realistic human exposure levels of ingestion were subjected to digestion simulation in a standardized in vitro static model and to gut-microbial dynamic fermentation using the simgi® model, with the aim of providing scientific evidence of modifications and potential effects of food MPs during their passage through the digestive tract. Furthermore, changes in microbiota composition and in PET morphology were evaluated after upper static digestion and after colonic fermentation in the different simgi® colonic compartments (ascending, transverse and descending colon).

\section{Results}




\section{Effect of gastrointestinal digestion and colonic fermentation in microplastics morphology}

The original PET MPs average particle size determined by image analysis was $160 \mu \mathrm{m} \pm 110 \mu \mathrm{m}$ and their morphology was irregular. The original PET MPs morphology and surface, which evidence a surface fracture of brittle material with multiple river marks, are shown in Fig. 1.a. During gastrointestinal digestion and colonic fermentations, the irregular morphology of the MPs is kept, as shown by FESEM micrographs (Fig. 1.a-i). After gastric (Fig. 1.c-d) and intestinal (Fig. 1.e-f) digestions, crystalline and organic matter deposits were observed on particle surfaces, especially after small intestinal digestion (Fig. 1.e), but no significant alteration of the polymer surface was observed. After dynamic in vitro colonic fermentations, however, an outstanding change of the PET MPs surfaces is observed (Fig. 1.g-i). The higher concentration of organic matter, proceeding from GNM and the faecal slurry, is deposited on the MPs surface (Fig. 1.g and i). Furthermore, images of Fig. 1.h-i show microbes on MPs surface adhered to the organic material deposits.

Raman spectra provide further molecular insight on the state of PET MPs. The Raman spectrum of PET presents the characteristic modes of polyethylene terephthalate, in line with known literature ${ }^{31}$. The spectral windows at $1780-1680$ and at $1220-1060 \mathrm{~cm}^{-1}$ Raman shift shown in Fig. 2 are of particular relevance to assess the crystalline order of PET ${ }^{32,33}$. Figure 2.a through 2.e show a progressive amorphization of PET. The Raman mode at $1727 \mathrm{~cm}^{-1}$ is associated with carbonyl stretching vibrations and is sensitive to the conformation of the terephthalate groups. In crystalline PET C = 0 groups are coplanar with an aromatic ring and in trans conformation with each other; these carbonyl groups arrange randomly with respect to the aromatic ring in amorphous PET ${ }^{34}$. A broadening of the band at $1727 \mathrm{~cm}^{-1}$ is the consequence of a loss of crystallinity, as illustrated in ${ }^{35}$. In addition, the Raman mode at $1186 \mathrm{~cm}^{-1}$ associated to the ring ester CCC bending in crystalline PET shifts to $1176 \mathrm{~cm}^{-1}$ in amorphous PET 32 . Cryomilling PET pellets to the original PET MPs results in a discrete broadening of $1727 \mathrm{~cm}^{-1}$ Raman band of the carbonyl and shift of the $1186 \mathrm{~cm}-$

${ }^{1}$ band of the ester, which are apparent at some spectra of PET MPs. The effect of stomach and intestine digestion is increasingly apparent on the states of PET MPs, where an increasing number of spectra show progressive amorphization. Such a trend is more evident after colonic fermentation, (Fig. 2.e). Further to these two Raman modes, the rise in the relative intensity of $1118 \mathrm{vs} .1094 \mathrm{~cm}^{-1}$ is also associated with oxidative amorphization of PET ${ }^{33}$. The relative intensity of 1118 modes vs. $1094 \mathrm{~cm}^{-1}$ is progressively higher as PET MP evolves along the different digestion stages, as observed in Fig. 2.f, which is consistent with the trends uncovered by the 1727 and $1186-1176 \mathrm{~cm}^{-1}$ Raman modes. This evolution indicates a trend in the structural degradation of the PET MPs during the gastrointestinal digestion.

\section{Impact of microplastics intervention on colonic microbiota}

The effect of PET MPs on the colonic microbiota in simgi® colonic compartments was evaluated by plate counting as a first approach (Fig. 3). From a microbiological point of view, differences between plate counting values were are considered significant for $\Delta \log (\mathrm{CFU} / \mathrm{mL}) \geq 1 \mathrm{due}$ to microbiological plate counting limitations ${ }^{22}$. Lactic acid bacteria, Enterococuss spp. and Staphylococcus spp. showed similar plate counting values during $72 \mathrm{~h}$ of colonic fermentation. On the contrary, total aerobic and total anaerobic bacteria decreased considerably in the three compartments $(\Delta$ $\log (\mathrm{CFU} / \mathrm{mL}) \geq 3)$, and Bifidobacterium spp. and Clostridium spp. followed the same trend, with a continuous reduction of viable bacteria in all three compartments of simgi ${ }^{\circledR}$. Finally, Enterobacteria group showed a decrease of $\Delta \log (\mathrm{CFU} / \mathrm{mL}) \geq 2$ in the first $24 \mathrm{~h}$ and a slightly lower decrease in the following $24 \mathrm{~h}$ in TC and DC compartments, while in AC the decrease was retarded. Between 48 and $72 \mathrm{~h}$ no significant changes were measured for the three compartments of the simulator.

Complementarily, 16S rRNA gene sequence analysis and subsequent study of microbial diversity were carried out. Regarding exposure to PET MPs, the alpha-diversity in terms of observed species and Shannon index decreased after the exposure in TC and DC compartments, but not in AC, which values remained stable for the $72 \mathrm{~h}$ of colonic fermentation (Fig. 4). Beta-diversity analysis revealed a grouping between AC samples at any time, while TC and DC samples showed a dispersion among $0 \mathrm{~h}$ and the other sampling times (24, 48 and $72 \mathrm{~h}$ ). This dispersion was more evident for TC samples, with higher differences on microbial profiles between all the analysed times, being these profiles after 48 and $72 \mathrm{~h}$ more similar to that observed for DC compartment at 24, 48 and $72 \mathrm{~h}$ (Fig. 5).

Deepening on the different taxonomic levels, phylogenetic analysis revealed differences in the proportions of some taxa after PET MPs intervention in the three colonic simgi ${ }^{\circledR}$ compartments. At phylum level, in general, the differences in the relative abundances of important taxa were colon compartment-dependent (Fig. 6). In AC compartment, Firmicutes and Desulfobacteria levels increased, while Bacteroidetes proportions decreased. For the TC, the Synergistetes, Proteobacteria and Desulfobacteria proportions rose, while the Bacteroidetes relative abundance strongly decreased up to below $10 \%$. In descending colon, Desulfobacteria phylum members duplicated their proportions in the first $24 \mathrm{~h}$, and Synergistetes levels were also incremented. On the contrary, the relative abundance of Bacteroidetes in DC showed an important decrease of more than $15 \%$ in the first $24 \mathrm{~h}$. Then the relative abundance remained stable until $72 \mathrm{~h}$ (Fig. 6).

The analysis of the microbiota key members at family level corroborated the results at phylum level, highlighting the decrease in Bacteroidaceae, Rikenellaceae and Tannerellaceae, and the increase in Lachnospiraceae, Enterobacteriaceae, Desulfovibrionaceae and Synergistaceae (Table 1). Regarding genus level, differences in the relative abundance of Bacteroides and Parabacteroides genus were revealed, with an important decrease in all colonic compartments, and particularly in TC and DC, where values below $5 \%$ were reached (Table 2). Supporting phyla analysis results, Proteobacteria members, such as Escherichia/Shigella and Bilophila, increased their levels in TC and DC, and in the case of Bilophila genus, also in 
AC. The proportions of Cloacibacillus, belonging to Synergistetes phylum, also increased in TC and DC, but were maintained in the AC compartment. Finally, Eisenbergiella, Megasphaera and Oscillibactergenus, belonging to Firmicutes phylum, increased their proportions in the ascending colon compartment, supporting the rise detected in this phylum after PET MPs intervention (Fig. 6).

Table 1

Evolution over time of the relative abundance at family level in each simgi® colonic compartment. Taxa with a relative abundance $>0.5 \%$ are represented.

\begin{tabular}{|c|c|c|c|c|c|c|c|c|c|c|c|c|c|}
\hline \multirow[b]{2}{*}{ Family } & \multirow[b]{2}{*}{ Time } & \multicolumn{4}{|c|}{ Ascending colon } & \multicolumn{4}{|c|}{ Transverse colon } & \multicolumn{4}{|c|}{ Descending colon } \\
\hline & & $\mathrm{Oh}$ & $24 \mathrm{~h}$ & $48 \mathrm{~h}$ & $72 \mathrm{~h}$ & $\mathrm{Oh}$ & $24 \mathrm{~h}$ & $48 \mathrm{~h}$ & $72 \mathrm{~h}$ & $\mathrm{Oh}$ & $24 \mathrm{~h}$ & $48 \mathrm{~h}$ & $72 \mathrm{~h}$ \\
\hline Acidaminococcaceae & & $1.1 \%$ & $0.3 \%$ & $0.2 \%$ & $0.5 \%$ & $3.3 \%$ & $1.4 \%$ & $1.0 \%$ & $0.6 \%$ & $3.6 \%$ & $1.6 \%$ & $1.9 \%$ & $0.6 \%$ \\
\hline Bacteroidaceae & & $57.7 \%$ & $56.2 \%$ & $56.2 \%$ & $46.9 \%$ & $36.1 \%$ & $18.4 \%$ & $5.9 \%$ & $4.3 \%$ & $17.9 \%$ & $7.4 \%$ & $7.6 \%$ & $5.0 \%$ \\
\hline Desulfovibrionaceae & & $3.5 \%$ & $5.2 \%$ & $5.9 \%$ & $13.3 \%$ & $5.5 \%$ & $10.3 \%$ & $15.5 \%$ & $14.4 \%$ & $9.2 \%$ & $18.3 \%$ & $18.5 \%$ & $20.8 \%$ \\
\hline Enterobacteriaceae & & $9.8 \%$ & $5.8 \%$ & $6.6 \%$ & $7.8 \%$ & $11.7 \%$ & $10.7 \%$ & $15.8 \%$ & $19.8 \%$ & $22.3 \%$ & $23.0 \%$ & $25.8 \%$ & $24.9 \%$ \\
\hline Lachnospiraceae & & $5.4 \%$ & $7.7 \%$ & $13.3 \%$ & $12.8 \%$ & $7.4 \%$ & $7.5 \%$ & $9.7 \%$ & $9.6 \%$ & $6.0 \%$ & $6.2 \%$ & $7.9 \%$ & $6.7 \%$ \\
\hline Oscillospiraceae & & $0.1 \%$ & $0.7 \%$ & $0.9 \%$ & $0.9 \%$ & $0.2 \%$ & $0.4 \%$ & $0.8 \%$ & $0.1 \%$ & $0.2 \%$ & $0.2 \%$ & $0.4 \%$ & $0.4 \%$ \\
\hline Oxalobacteraceae & & $0.0 \%$ & $0.0 \%$ & $0.0 \%$ & $0.0 \%$ & $0.1 \%$ & $0.5 \%$ & $1.0 \%$ & $0.9 \%$ & $0.3 \%$ & $0.5 \%$ & $0.6 \%$ & $0.5 \%$ \\
\hline Peptostreptococcaceae & & $0.0 \%$ & $0.0 \%$ & $0.0 \%$ & $0.0 \%$ & $1.0 \%$ & $0.2 \%$ & $0.0 \%$ & $0.0 \%$ & $0.5 \%$ & $0.0 \%$ & $0.0 \%$ & $0.0 \%$ \\
\hline Rikenellaceae & & $2.8 \%$ & $1.5 \%$ & $0.5 \%$ & $0.5 \%$ & $4.8 \%$ & $11.4 \%$ & $4.3 \%$ & $1.0 \%$ & $2.5 \%$ & $0.8 \%$ & $0.2 \%$ & $0.9 \%$ \\
\hline Sutterellaceae & & $0.0 \%$ & $0.0 \%$ & $0.0 \%$ & $0.0 \%$ & $0.7 \%$ & $1.7 \%$ & $2.2 \%$ & $2.0 \%$ & $1.0 \%$ & $1.0 \%$ & $1.0 \%$ & $0.8 \%$ \\
\hline Synergistaceae & & $5.7 \%$ & $9.1 \%$ & $8.6 \%$ & $8.0 \%$ & $14.6 \%$ & $26.0 \%$ & $36.2 \%$ & $42.8 \%$ & $29.5 \%$ & $39.2 \%$ & $34.9 \%$ & $38.3 \%$ \\
\hline Tannerellaceae & & $12.5 \%$ & $12.4 \%$ & $5.8 \%$ & $4.4 \%$ & $12.4 \%$ & $4.7 \%$ & $2.0 \%$ & $1.2 \%$ & $5.1 \%$ & $0.5 \%$ & $0.3 \%$ & $0.0 \%$ \\
\hline Veillonellaceae & & $1.3 \%$ & $0.9 \%$ & $1.8 \%$ & $4.1 \%$ & $1.1 \%$ & $3.5 \%$ & $4.6 \%$ & $1.9 \%$ & $0.7 \%$ & $0.2 \%$ & $0.1 \%$ & $0.0 \%$ \\
\hline
\end{tabular}


Table 2

Evolution over time of the relative abundance at genus level in each simgi® colonic compartment. Taxa with a relative abundance $>0.5 \%$ are represented.

\begin{tabular}{|c|c|c|c|c|c|c|c|c|c|c|c|c|c|}
\hline \multirow[b]{2}{*}{ Genus } & \multirow[b]{2}{*}{ Time } & \multicolumn{4}{|c|}{ Ascending colon } & \multicolumn{4}{|c|}{ Transverse colon } & \multicolumn{4}{|c|}{ Descending colon } \\
\hline & & $\mathrm{Oh}$ & $24 \mathrm{~h}$ & $48 \mathrm{~h}$ & $72 \mathrm{~h}$ & $\mathrm{Oh}$ & $24 \mathrm{~h}$ & $48 \mathrm{~h}$ & $72 \mathrm{~h}$ & $\mathrm{Oh}$ & $24 \mathrm{~h}$ & $48 \mathrm{~h}$ & $72 \mathrm{~h}$ \\
\hline Alistipes & & $2.8 \%$ & $1.5 \%$ & $0.5 \%$ & $0.5 \%$ & $4.8 \%$ & $11.4 \%$ & $4.3 \%$ & $1.0 \%$ & $2.5 \%$ & $0.8 \%$ & $0.2 \%$ & $0.9 \%$ \\
\hline Bacteroides & & $57.7 \%$ & $56.2 \%$ & $56.2 \%$ & $46.9 \%$ & $36.1 \%$ & $18.4 \%$ & $5.9 \%$ & $4.3 \%$ & $17.9 \%$ & $7.4 \%$ & $7.6 \%$ & $5.0 \%$ \\
\hline Bilophila & & $3.0 \%$ & $4.7 \%$ & $5.3 \%$ & $13.0 \%$ & $4.8 \%$ & $9.8 \%$ & $14.8 \%$ & $13.9 \%$ & $8.7 \%$ & $17.5 \%$ & $17.6 \%$ & $20.1 \%$ \\
\hline Cloacibacillus & & $5.7 \%$ & $9.1 \%$ & $8.6 \%$ & $8.0 \%$ & $14.6 \%$ & $26.0 \%$ & $36.2 \%$ & $42.8 \%$ & $29.5 \%$ & $39.2 \%$ & $34.9 \%$ & $38.3 \%$ \\
\hline Coprococcus & & $0.3 \%$ & $0.5 \%$ & $0.9 \%$ & $0.6 \%$ & $0.1 \%$ & $0.0 \%$ & $0.0 \%$ & $0.0 \%$ & $0.0 \%$ & $0.0 \%$ & $0.0 \%$ & $0.0 \%$ \\
\hline Desulfovibrio & & $0.4 \%$ & $0.5 \%$ & $0.6 \%$ & $0.3 \%$ & $0.6 \%$ & $0.5 \%$ & $0.7 \%$ & $0.5 \%$ & $0.5 \%$ & $0.9 \%$ & $0.9 \%$ & $0.8 \%$ \\
\hline Eisenbergiella & & $3.0 \%$ & $3.1 \%$ & $5.5 \%$ & $8.5 \%$ & $2.2 \%$ & $5.4 \%$ & $7.0 \%$ & $7.2 \%$ & $1.3 \%$ & $2.9 \%$ & $3.6 \%$ & $2.8 \%$ \\
\hline Enterobacter & & $0.3 \%$ & $0.3 \%$ & $0.3 \%$ & $0.3 \%$ & $0.4 \%$ & $0.7 \%$ & $0.6 \%$ & $0.4 \%$ & $0.4 \%$ & $0.3 \%$ & $0.1 \%$ & $0.2 \%$ \\
\hline Escherichia/Shigella & & $7.5 \%$ & $4.5 \%$ & $4.9 \%$ & $5.7 \%$ & $7.4 \%$ & $6.1 \%$ & $10.1 \%$ & $13.5 \%$ & $13.3 \%$ & $15.5 \%$ & $16.7 \%$ & $15.6 \%$ \\
\hline Intestinimonas & & $0.0 \%$ & $0.0 \%$ & $0.0 \%$ & $0.0 \%$ & $0.2 \%$ & $0.4 \%$ & $0.7 \%$ & $0.1 \%$ & $0.1 \%$ & $0.2 \%$ & $0.4 \%$ & $0.4 \%$ \\
\hline Klebsiella & & $1.3 \%$ & $0.2 \%$ & $0.1 \%$ & $0.2 \%$ & $1.5 \%$ & $0.1 \%$ & $0.0 \%$ & $0.0 \%$ & $0.7 \%$ & $0.5 \%$ & $0.4 \%$ & $0.4 \%$ \\
\hline Lachnoclostridium & & $0.6 \%$ & $0.7 \%$ & $0.5 \%$ & $0.5 \%$ & $1.3 \%$ & $1.1 \%$ & $1.7 \%$ & $1.5 \%$ & $2.2 \%$ & $2.6 \%$ & $3.4 \%$ & $2.8 \%$ \\
\hline Megasphaera & & $1.3 \%$ & $0.9 \%$ & $1.8 \%$ & $4.1 \%$ & $1.1 \%$ & $3.5 \%$ & $4.6 \%$ & $1.9 \%$ & $0.7 \%$ & $0.2 \%$ & $0.1 \%$ & $0.0 \%$ \\
\hline Oscillibacter & & $0.1 \%$ & $0.2 \%$ & $0.4 \%$ & $0.6 \%$ & $0.0 \%$ & $0.0 \%$ & $0.0 \%$ & $0.0 \%$ & $0.1 \%$ & $0.0 \%$ & $0.0 \%$ & $0.0 \%$ \\
\hline Oxalobacter & & $0.0 \%$ & $0.0 \%$ & $0.0 \%$ & $0.0 \%$ & $0.1 \%$ & $0.5 \%$ & $1.0 \%$ & $0.9 \%$ & $0.3 \%$ & $0.5 \%$ & $0.6 \%$ & $0.5 \%$ \\
\hline Parabacteroides & & $12.3 \%$ & $12.1 \%$ & $5.6 \%$ & $4.3 \%$ & $12.0 \%$ & $4.6 \%$ & $1.9 \%$ & $1.2 \%$ & $4.8 \%$ & $0.5 \%$ & $0.3 \%$ & $0.0 \%$ \\
\hline Parasutterella & & $0.0 \%$ & $0.0 \%$ & $0.0 \%$ & $0.0 \%$ & $0.7 \%$ & $1.7 \%$ & $2.2 \%$ & $2.0 \%$ & $1.0 \%$ & $1.0 \%$ & $1.0 \%$ & $0.8 \%$ \\
\hline Phascolarctobacterium & & $1.1 \%$ & $0.3 \%$ & $0.2 \%$ & $0.5 \%$ & $3.3 \%$ & $1.4 \%$ & $1.0 \%$ & $0.6 \%$ & $3.6 \%$ & $1.6 \%$ & $1.9 \%$ & $0.6 \%$ \\
\hline Terrisporobacter & & $0.0 \%$ & $0.0 \%$ & $0.0 \%$ & $0.0 \%$ & $1.0 \%$ & $0.2 \%$ & $0.0 \%$ & $0.0 \%$ & $0.5 \%$ & $0.0 \%$ & $0.0 \%$ & $0.0 \%$ \\
\hline
\end{tabular}

\section{Discussion}

Our study demonstrates that microplastic feeding affects both composition and diversity of colonic microbial communities. To date, the studies on this field have been focused on the effect of MPs on the gut microbial communities of soil animals or mice 14,15,36,37, and to our knowledge, this is the first report about the modifications and potential effects of microplastics on human colonic microbiota. Moreover, the doses of MPs tested in previous studies with animal models are higher than those detected in edible foods and beverages, but lower than the estimated human daily intake ${ }^{14,16,36,38-}$

42. Our approach was to expose human colonic microbiota to a concentration of microplastics closer to reality, so we selected 166 mg/intake of PET MPs, corresponding to the estimated daily intake in humans, according to the study of Senathiraja et al. ${ }^{3}$. We focused our study on the changes during the gastrointestinal digestion and fermentation processes both of PET microparticles' morphology and structure and of the colonic microbial communities responsible for metabolic bioconversions in the large intestine. For this reason, PET MPs underwent different treatments to simulate the oral, gastric and small intestinal phases of the digestion before getting in contact with previously stabilized human colonic microbiota in the simgi® dynamic simulator, which mimicked the overall impact of digested microplastics on the complex microbial intestinal ecosystem ${ }^{19,25}$.

PET thermoplastic, as well as other polymers materials, show viscoelastic behavior during grinding, which results in a plastic deformation. This inhibited crack initiation, and hence a break-up did not happen. Thus, the PET pellets were grinded in liquid nitrogen, and this allowed cracking them into smaller particles of ca. $60 \mu \mathrm{m}$. PET MPs surface evidenced the appearance of brittle fractures with abundant river marks, associated with the compression side during cryogrinding, and few ductile fracture regions. In agreement, the Raman spectra of the MPs suggest a slight loss of crystallinity with respect to the net pellet. Regarding the effect of gastrointestinal digestion and colonic fermentation on PET MPs, FESEM images revealed an evolution of the surface after in vitro intestinal digestion, indicative of a slight interaction with the media, which resulted, according to Raman characterization, with a relative amorphization of the PET structure, but no remarkable morphological changes. PET MPs average size was large enough to avoid disintegration during digestion, allowing us to monitor the MPs at the different intestinal regions. Salt and organic matter deposits were observed on the particle surfaces after gastrointestinal digestion, as previously reported in the work of Stock et al ${ }^{12}$. Crystalline deposits were more abundant, fact that can be related to the increase in salts concentration due to simulated gastric fluid (SGF) and simulated 
intestinal fluid (SIF) addition during gastrointestinal digestion. Besides, some organic deposits, probably forming the so-called (protein-) corona ${ }^{12}$, were also found on PET MPs surfaces at this point, possibly due to the presence of enzymes from the digestion simulation. MPs morphologies show the same appearance after gastrointestinal digestion agreeing with Stock ${ }^{12}$ results, despite particle size differences. It has been suggested that the particles' size, more than their chemical structure, as well as their possible deformation or degradation, and the protein corona formation during the digestive process are crucial for the bioavailability, and thus for the intestinal uptake rate of the particles and the subsequent toxicological impact and health risks ${ }^{12}$.

After colonic fermentation, PET MPs presented remarkable organic deposits on their surface, which seemed to be colonized by some members of the colonic microbiota, and the surface roughness evolved towards a globular surface. Moreover, the crystalline structure of the PET MPs is affected after the colonic fermentation, which confirms the biotransformation of the polymer by the human digestion process already observed after the gastric step. The FESEM micrographs show in some cases damaged or broken bacteria adhered to the MPs surface, not allowing to observe an intact microbiota. This may be due to the fact that the cells were not subjected to a fixation process just after sampling and prior to the treatment for their observation in the FESEM, so as not to alter the structure of the PET MPs. Even so, different adhered bacterial cells and biofilm-like structures were observed in colonic samples, supporting the hypothesis that some members of the colonic microbiota could adhere to MPs surface. In relation to the MPs colonization, different human gut microbial species, such as Escherichia coli, Pseudomonas aeruginosa and Staphylococus epidermidis, have shown the ability to adhere and even form biofilms on diverse plastic material surfaces such as polyethylene, polypropylene and polystyrene, among others ${ }^{8,43,44}$. Besides, a recent work has shown the adhesion of the gut microbiota of honey bees to the surface of polystyrene MPs ${ }^{45}$. Bacterial communities present on colonic microbiota adhere and colonize the gut mucosa, forming biofilms essential to their cross-feeding relationships, nutrient availability and protection against toxins (i.e. antibiotics), mechanical damage and shear caused by fluid flow 43 . In our study, the possible adhesion of some members of the colonic microbiota to the PET MPs surface could be due to the absence of a mucus layer or intestinal epithelium to adhere to in the simgi®, promoting the formation of biofilms on MPs with the aim to protect themselves and establish their relationships and functions as a community. Another possibility could be the adhesion of some bacterial species able to metabolize and degrade the PET MPs. In this regard, although it has been reported that PET is resistant to biodegradation, various bacterial hydrolases from environmental samples, such as cutinases, lipases, carboxylesterases, and esterases, have been shown to degrade PET to different extents ${ }^{46}$. In general, polymer biodegradation processes include different degrees of material decomposition, which can start with non-enzymatic hydrolysis that promotes fragmentation, followed by assimilation by microorganisms that further involves the enzymatic degradation ${ }^{47}$. In this sense, the Ideonella sakaiensis PETase depolymerizes $\mathrm{PET}$, liberating soluble products ${ }^{48}$. Besides, some gut bacterial strains from earthworms have displayed the ability to reduce significantly the particle size of low-density polyethylene ${ }^{44}$. In our work, Raman results showed progressive amorphization of PET MPs during gastrointestinal digestion, possibly related to oxidative amorphization of PET ${ }^{33}$, and more evident after colonic fermentation. These structural changes in the PET MPs particles suggest a potential biodegradation probably driven by colonic microbiota, supporting the existence of an interaction between the colonic microbiota and PET MPs particles. However, although we cannot rule out the presence of PET degrading activities in some specific members of the human colonic microbiota, so far there are no studies or evidence of the existence of human gut bacterial species able to degrade MPs.

Further research is needed to analyze the possible PET MPs colonization by members of human colonic microbiota after ingestion and the mechanisms and functions underlying this adhesion, as well as to explore the possible presence of bacterial activities on human gut microbiome susceptible to biodegrade PET MPs or affect its morphology and structure. Additionally, longer duration experiments should be performed to observe if bacteria from colonic microbiota can degrade plastic over time when faced with a labile $\mathrm{C}$ limitation. Another factor for future consideration is the comparison with different MPs polymers and sizes, and the potential degradation depending on the polymer type. Moreover, in the present study a net PET without polymer processing additives was used. The effect of such polymer additives must be also further evaluated because are currently used in commercial products.

Regarding the effect of the intervention with PET MPs on the colonic microbial communities stabilized in simgi ${ }^{\circledR}$, plate counts and relative abundances of different bacterial groups revealed changes after $72 \mathrm{~h}$ of fermentation. The microbiota has undergone a stabilization process prior to the intervention for 14 days followed to a control period of 5 days $(120 \mathrm{~h})$ with stable microbial populations presenting a microbial profile of the similar to time $0 \mathrm{~h}$ of PET MPs exposure, so we assume that without any disturbance, colonic microbiota should maintain their levels and remain stable for the $72 \mathrm{~h}$ of PET MPs exposure. This fact is supported by bacterial counts results, that showed stable levels of different bacterial groups as lactic acid bacteria, Staphylococcus and Enterococcus during the $72 \mathrm{~h}$.

Bacterial counts show reductions in total aerobic and anaerobic bacteria, as well as in Bifidobacterium spp. and Clostridium spp. This suggests that PET MPs and/or their potential resulting constituent monomers exert a negative effect against colonic microbiota, decreasing the levels of total viable bacteria, and to a greater extent of certain microbial groups such as Bifidobacterium, Clostridium and enterobacteria. Even though the bacterial counts method is widely used in simulators to routinely monitor how the colonic microbiota is evolving during the process, this technique provides information only about the viable and cultivable bacteria, allowing to detect about $20 \%$ of the present microbial communities ${ }^{49}$. Hence, it is not the best approximation to deepen into the real microbial changes produced in response to the intervention with PET MPs in simgi®. Accordingly, $16 \mathrm{~S}$ rRNA gene metagenomic analysis was performed to delve in the microbial communities' changes, obtaining different trends for the bacterial biodiversity and for different taxonomic levels. 
PET MPs intervention revealed changes in the biodiversity and the relative abundances of different taxa in the three compartments of simgi ${ }^{\circ}$. Regarding biodiversity, PET MPs promote a decrease in the alpha diversity indices in terms of Observed species and Shannon index, more evident for the TC and DC compartments. This decrease in the alpha biodiversity indices has been previously reported in animal models after a exposure to MPs, as well as the changes in the beta diversity $14,38,39,41,45$, indicating that the structure of the gut microbiota is altered after MPs exposure. Bacteroidetes levels showed an important decrease after PET MPs intervention, being this effect more powerful in the case of TC and DC compartments, reaching levels below $5 \%$ of relative abundance. This result also agrees with those reported in other intervention studies with MPs in different animal models that detected an important drop in the relative abundance of the members of this phylum ${ }^{16,38,39,41}$. This trend is also supported by our results at family and genus levels, detecting lower levels of Bacteroides and Parabacteroides, in agreement with other studies ${ }^{50,51}$, and suggesting that MPs could have an important antibacterial effect on these key members of the gut microbiota. Bacteroides and Parabacteroides species include many important opportunistic pathogens, but as essential members of a balanced microbiota, they are considered to be health-maintaining. These two groups have the ability to reinforce the epithelial barrier and ameliorate inflammation by producing anti-inflammatory molecules such as polysaccharide A (PSA), sphingolipids and outer membrane vesicles (OMVs) for the transport of these molecules to the epithelium, and produce antibacterial molecules to prevent the colonization and invasion of exogenous bacteria 52,53 . So, the decrease detected in the proportions of these taxa in vitro after PET MPs intervention is expected be reproduced in vivo in the human gut, which would mean the decrease or even loss of two essential groups not only for the maintenance of the correct balance of the gut microbial communities, but also for the intestinal immune homeostasis and barrier function, which imbalance has been associated with systemic and intestinal diseases, such as inflammatory bowel disease (IBD) and irritable bowel syndrome (IBS), among others ${ }^{52,54}$. Firmicutes phylum members increased in the AC compartment, although this trend was not detected for the other two colonic compartments. The increase in the proportions of Firmicutes phyla after MPs intervention is a trend previously reported in other animal models such as zebrafish and mice $16,38,39,41$. The changes in the proportions of these two phyla promotes an increment in Firmicutes/Bacteroidetes ratio, known to be of significant relevance for the human gut microbiota status, and often used as a biomarker in connection with human physiology, being its imbalance related to different metabolic disorders such as obesity and diabetes ${ }^{55-58}$. Moreover, the increase detected in Proteobacteria proportions in the TC and DC compartments also agrees with other studies in different animal models ${ }^{16,40,42}$, being in our work this rise mainly driven by the increase in the proportions of Escherichia/Shigella and Bilophila genus members. Although a decrease in bacterial counts of Enterobacteria group is detected, 16S rRNA gene metagenomic analysis revealed an increase in the proportions of Enterobacteriaceae family and Escherichia/Shigella genus members. These techniques provide different approaches, not comparable but complementary, being bacterial counts a quantitative approach and the 16S rRNA sequencing a relative abundance result relative to the total number of microorganisms. In addition, the 16S rRNA gene analysis is capable of detecting not only cultivable microorganisms, but also microbial groups that cannot be cultivated under laboratory conditions, providing an image more complete and closer to the real situation. Bearing this in mind, this contradictory result could be due to the fact that despite a decrease in the total counts of members of this group is detected, also observed for the total of aerobes and anaerobes in general, there seems to be an increase in their relative proportion within the microbiota as a whole. A similar effect was also reported by Lu and colleagues, that observed a decrease in a-Proteobacteria levels by qPCR, and an increase in their relative abundance by $16 \mathrm{~S}$ rRNA sequencing ${ }^{15}$. Proteobacteria is a microbial signature of inflammation in the gut ${ }^{59}$, and some members belonging to this phylum, such as Escherichia/Shigella and Bilophila, have been widely associated with a pro-inflammatory effect, as for example Bilophila wadsworthia, that promotes pro-inflammatory TH1 immunity and exacerbates colitis in IBD-prone II10-/- mice ${ }^{60}$. The rise in the proportions of these pro-inflammatory bacterial groups has been related to different diseases, such as IBD, colorectal cancer, and coronary artery disease ${ }^{61-64}$. Finally, Synergistetes phylum also reveals an important increase in response to PET MPs exposure in simgi®, being the rise in Cloacibacillus genus the main responsible of this effect. Members belonging to this genus have been detected in elevated levels in patients with type 2 diabetes, colorectal cancer and even in Parkinson's disease ${ }^{65-67}$, and have also been positively correlated to a pro-inflammatory effect 68 .

In summary, our results reveal that the exposure of the human colonic microbiota to PET MPs clearly affected the microbial communities present, as reported for other micro- and nanoplastics $13,37,69$, and other nanoparticles ${ }^{29}$. This might negatively affect human health, by: i) the decrease of microbial groups essential for the correct balance of the gut microbiota and for the intestinal immune homeostasis and barrier function; and ii) the increase of different well-known pro-inflammatory and disease-related bacterial groups, fact that could lead to a chronic gut inflammation state, damaging the epithelial barrier and intestinal permeability, and increasing the risk of developing not only local diseases, such as IBD, but also systemic disorders. Besides, this work is the first study that evaluates the potential bidirectional effect of the PET MPs in an in vitro standardized gastrointestinal digestion static model and gut-microbial dynamic fermentation. The simgi® simulator allows to recreate and control in vitro a situation closer to reality than other in vitro models in terms of physiological conditions, human gastrointestinal microbiota populations and metabolic activity, providing a more real image of the interactions between PET MPs and human gut microbial communities. Our findings agree with trends reported in animal models that analyse the effect of different MPs on gut microbial communities, and have biological relevance from a microbial and health point of view. However, to date there are only a few works focused on this field, and none of them has been carried out in a gastrointestinal simulator that allows to mimic the physiological conditions of human gut microbiome and digestion process, fact that makes it difficult to compare the results. Furthermore, our data are not enough to perform a powerful statistical analysis. Nevertheless, even considering these limitations, our results suggest a clear interaction between the PET MPs and the human colonic microbiota, and establish a knowledge basis of the potential PET MPs effects on the bacterial communities present in the human intestine, and the possible effect of these microbial populations on PET MPs, useful for future investigations oriented to unravelling the real effect of these environmental contaminants on human health. 
On the other hand, considering that the presence of microplastics is generalized and that the amount and variety of microplastics in the environment may rise exponentially with the massive increase in the use of polymers ${ }^{70}$, initiatives to diminish the utilization of single-use plastics are important to reduce their impact. However, the vast majority of plastics are far from a circular economy process that would undoubtedly minimize the uncontrolled increase of microplastics in the environment. This study, which considered the impact of PET primary MPs, covered only one of the key possible impact points of MP ingestion on gut microbiota. Other critical aspect is the large number of process additives present in polymers, with unsuspected effects on gut microbiota. Polymer materials used in food packaging follow a strict regulation ${ }^{71}$ that authorizes just hundreds of polymer additives, but those that are prohibited for food packaging, that exceed the number of approved ones, are used in polymers for other applications that could partly end up as MPs and form part of the diet, albeit undesirably. Moreover, MP ingestion could generate additional risk factors, since MPs coming from the environment could also act as vectors for possible pathogens or contaminants, which could directly or indirectly impact in gut microbiota and be related with gut dysbiosis ${ }^{13,72}$. Hence, further research is needed to elucidate the effect of MPs intake on the human gut microbiome homeostasis, and thus be able to assess the risk that MPs ingestion through diet has on human health.

\section{Methods}

The experimental set-up is showed schematically in Fig. 7.

\section{Microplastics origin and characterization}

Net PET was used without additional processing additives that could distort crystallinity or chemical reactivity. Pure polymer pellets of polyethylene terephthalate (Ramapet N80, Indorama Polymers Europe, Lithuania) were blade milled in liquid nitrogen to obtain PET microparticles. PET MPs and samples from gastrointestinal digestion and colonic fermentations were gold coated $(\approx 200 \AA)$ to prepare samples for size and morphology examination by field emission scanning electron microscopy (FESEM) (Hitachi S-4700, Hitachi, Japan) at 20 keV. Optical analysis was performed in a Multimode Optical Profilometer (Zeta-20, Zeta Instruments, USA). The structural changes of the PET MPs after the gastrointestinal digestion and colonic fermentation were evaluated by micro-Raman in a Renishaw Qontor spectrometer with a $514 \mathrm{~nm}$ excitation line laser. The spectral resolution is $1.4 \mathrm{~cm}^{-1}$ and every sample was analyzed running a Raman map on at least 60 points to generate representative sampling of the state of PET samples. Each spectrum consisted of 60 accumulations of 1 second with a laser power or $1.5 \mathrm{~mW}$ and a $50 \mathrm{x}$ microscope objective. This allows micron-sized space resolution. The total laser power density was low enough to avoid laser damage on the sample during mapping.

\section{In vitro static gastrointestinal digestions}

The selected PET MPs dose for gastrointestinal digestion was $0.166 \mathrm{~g} /$ intake, which corresponds to the estimated mean of MPs ingest for humans. Senathiraja et al. assessed that humans may ingest between 0.014 and $0.714 \mathrm{~g}$ of MPs daily ( 0.1 to $5 \mathrm{~g}$ per week) ${ }^{3}$. If we consider $4-5$ intakes per day, then the intake has a maximum of $0.143-0.179 \mathrm{~g}$.

Gastrointestinal digestions of MPs were performed by INFOGEST method ${ }^{73}$. Briefly, to simulate the oral phase, the MPs were suspended in Milli-Q water and mixed with simulated salivary fluid (SSF). The mixtures were incubated in an orbital shaker for 2 min at $37^{\circ} \mathrm{C}$ to simulate oral step, then simulated gastric fluid (SGF) and commercial porcine pepsin were added, providing an enzymatic activity of $2000 \mathrm{U} / \mathrm{mL}$ in the final digestion mixture. The $\mathrm{pH}$ was adjusted to 3 , and the samples were incubated for $2 \mathrm{~h}$ at $37^{\circ} \mathrm{C}$. After the gastric stage, simulated intestinal fluid (SIF) was added and the digested samples were set to $\mathrm{pH}$ 7. Finally, the intestinal phase was simulated by adding bile salts and pancreatin in order to reach $100 \mathrm{U} / \mathrm{mL}$ of trypsin activity and $10 \mathrm{mM}$ of bile salts in the final mixture, which was incubated in the same conditions for 2 more hours. To stop the digestion process, the samples were immediately frozen at $-80^{\circ} \mathrm{C}$ (for $24 \mathrm{~h}$ ) and kept at $-20^{\circ} \mathrm{C}$ until posterior use in colonic fermentations.

\section{In vitro colonic fermentation using simgi ${ }^{\circledR}$}

Colonic fermentations using human colonic microbiota were carried out in simgi® system.

Simgi ${ }^{\circledR}$ is a computer-controlled dynamic simulator of the gastrointestinal tract consisting on five successive reactors able to reproduce gastrointestinal digestion and colonic fermentation ${ }^{19,25}$. In the present study, simgi® system modular design was used to simulate the tree regions of the human colon: ascending (AC), transverse (TC) and descending (DC) colon. The reactors were kept at $37^{\circ} \mathrm{C}$ and $150 \mathrm{rpm}$ in anaerobic condition. By automatic addition of $0.5 \mathrm{NaOH}$ and $\mathrm{HCl}, \mathrm{pH}$ and volumes were maintained at 5.6 and $250 \mathrm{~mL}$ for $\mathrm{AC}, 6.3$ and $400 \mathrm{~mL}$ for TC, and 6.8 and $300 \mathrm{~mL}$ for $\mathrm{DC}$, respectively. Flow rates, compartment volumes, $\mathrm{pH}$, temperature, and pressure and transferences were computer controlled during the experiments.

The system set-up was conducted according to the scheme of Fig. 7. The process started with the stabilization of the colonic microbiota in the colonic reactors (AC, TC and DC). For this, the three compartments were inoculated with a $20 \%(\mathrm{w} / \mathrm{v})$ fresh faecal suspension of a healthy volunteer (faecal inoculum) with no history of gastrointestinal disorders or antibiotic treatment for at least 3 months before the study, as described in Tamargo et al. ${ }^{74}$. Then, the system remained in operation for 14 days for the stabilization of the microbiota, as previously described ${ }^{19}$. During the microbiota 
stabilization step, the system was fed every $8 \mathrm{~h}$ with $75 \mathrm{~mL}$ of Gut Nutrient Medium (GNM) containing arabinogalactan (1 g/L), citrus peel pectin (2 $\mathrm{g} / \mathrm{L})$, xylan $(1 \mathrm{~g} / \mathrm{L})$, potato starch $(3 \mathrm{~g} / \mathrm{L})$, glucose $(0.4 \mathrm{~g} / \mathrm{L})$, yeast extract $(3 \mathrm{~g} / \mathrm{L})$, peptone $(1 \mathrm{~g} / \mathrm{L})$, mucin $(4 \mathrm{~g} / \mathrm{L})$, and L-cysteine $(0.5 \mathrm{~g} / \mathrm{L})$. Once the microbiota was stabilized, each colonic compartment was fed with a single dose of digested PET MPs ( $0.166 \mathrm{~g})$ and the conditions were kept for 72 h. Samples from all compartments (AC, TC and DC) were specifically collected before PET MPs feeding ( $0 \mathrm{~h})$, and after 24,48 and $72 \mathrm{~h}$. Microbial plate count analyses were performed at the time of the sample collection, while the samples were stored at $-80^{\circ} \mathrm{C}$ until further analyses were carried out. Experimental protocol with the faecal human sample was approved by the Ethics Committee of The Spanish National Research Council (CSIC) being assessed under the internal registration code AGL2015-64522, and was compliant with the Declaration of Helsinki. Signed informed consent from donors data was obtained at the time of enrolment.

\section{Colonic microbiota analysis}

\section{Plate counting}

Immediately after sampling, ten-fold serial dilutions of AC, TC and DC contents were plated on different types of selective media as described in Tamargo et al. ${ }^{74}$. Briefly: Trypticase Soy Agar (TSA) (Difco ${ }^{\mathrm{TM}}, \mathrm{BD}, \mathrm{USA}$ ) was used as media for total aerobes plate counting; Wilkins Chalgren agar (Difco $\left.{ }^{\mathrm{TM}}, \mathrm{BD}\right)$ for total anaerobes; MacConkey agar (Difco $\left.{ }^{\mathrm{TM}}, \mathrm{BD}, \mathrm{USA}\right)$ for Enterobacteriaceae, Enterococcus agar (Difco $\left.{ }^{\mathrm{TM}}, \mathrm{BD}, \mathrm{USA}\right)$ for Enterococcus spp.; MRS agar ( $\mathrm{pH}=5.4$ ) (Pronadisa, CONDA, Spain) for lactic acid bacteria; Tryptose Sulfite Cycloserine agar (TSC) (Pronadisa, CONDA, Spain) for Clostridium spp.; BBL CHROMAgar Staph aureus (Difco ${ }^{\mathrm{TM}}, \mathrm{BD}, \mathrm{USA}$ ) for Staphylococcus spp.; Bifidobacterium agar modified by Beerens (Difco ${ }^{\mathrm{TM}}$, BD, USA) for Bifidobacterium spp.; and LAMVAB for specific faecal Lactobacillus spp. All plates were incubated at $37^{\circ} \mathrm{C}$ for 24 to $72 \mathrm{~h}$ in anaerobic conditions (BACTRON Anaerobic Environmental Chamber, SHELLAB, USA), except for BBL CHROMAgar Staph aureus and TSA, which were incubated in aerobic conditions. Plate counting was done by triplicate and data are expressed as $\log (\mathrm{CFU} / \mathrm{mL})$.

\section{DNA extractions from colonic samples and Illumina MiSeq sequencing}

$2 \mathrm{~mL}$ samples from AC, TC and DC simgi® compartments were used for DNA extraction using the QIAamp DNA Stool Mini Kit (Qiagen, Hilden, Germany). The V3-V4 region of the 16S ribosomal RNA gene was amplified using forward 5'-CCTACGGGNBGCASCAG-3' and reverse 5'-

GACTACNVGGGTATCTAATCC-3' primers. The two-step Illumina ${ }^{\circledR}$ PCR protocol was followed to prepare the libraries, and samples were submitted to $2 \times 500$ bp paired-end sequencing by means of an Illumina ${ }^{\circledR}$ MiSeq instrument (Illumina ${ }^{\circledR}$, USA). RStudio v.1.3.1093 software was used to process the files with raw reads from Illumina ${ }^{\circledR}$ instrument. The fastqc files were filtered for reads with low quality and presence of alien DNA using DADA2. DADA2 algorithm was also employed to denoise, join paired-end reads and filter out chimeras in the raw data ${ }^{75,76}$. This algorithm allows the differentiation of even a single nucleotide, leading to the formation of Amplicon Sequence Variants (ASVs). The taxonomic assignment was performed using the naïve Bayesian classifier implemented in DADA2 using Silva v.138 as reference database ${ }^{77}$, with a bootstrap cut-off of $80 \%$. A total of 166 ASVs were found. Biodiversity, expressed in terms of alpha-diversity, was estimated using the ASVs by calculating the Observed, Shannon and Simpson indices through the "Phyloseq" package. Beta-diversity was evaluated employing a Bray-Curtis dissimilarity matrix represented by nonmetric multidimensional scaling (NMDS).

\section{Declarations}

\section{Acknowledgements}

This research was funded by the Spanish Ministry of Science and Innovation (Spain), grants number AGL2015-64522-C2-1-R and PID2019-108851RBC21, and ALIBIRD-CM 2020 P2018/BAA-4343 (Community of Madrid).

\section{Author contributions}

A.T., M.A.B., J.F.F. and M.V.M.A. conceived the experiment(s); J.J.R. and J.F.F. produced microplastics and performed FESEM analysis; V.A.-R., R.P. and M.A.B. performed Raman experiments; A.T., N.M., and M.V.M.A. designed and conducted gastrointestinal simulation experiments and microbiome analysis; A.T., N.M, and M.V.M.A. analysed the results. All authors contributed to the writing, editing, and review of the manuscript.

\section{COMPETING INTERESTS}

The authors declare no competing interests.

\section{References}

1. Cox, K. D. et al. Human Consumption of Microplastics. Environ. Sci. Technol. 53, 7068-7074 (2019).

2. Paul, M. B. et al. Micro- And nanoplastics-current state of knowledge with the focus on oral uptake and toxicity. Nanoscale Advances 2 , 43504367 (2020). 
3. Senathirajah, K. et al. Estimation of the mass of microplastics ingested - A pivotal first step towards human health risk assessment. J. Hazard. Mater. 404, 124004 (2021).

4. Tamargo, A., Jiménez-Gómez, M. B., Bartolomé, B., Cueva, C., \& Moreno-Arribas, M. V. Micro and nanoplastics: from food occurrence to impact on human gastrointestinal health. Trends Food Sci. Technol. under revi, (2021).

5. EFSA. Initial Statement by the Group of Chief Scientific Advisors: A Scientific Perspective on Microplastic Pollution and its Impacts. Eu Publ. 1-4 (2018).

6. Presence of microplastics and nanoplastics in food, with particular focus on seafood. EFSA J. 14, e04501 (2016).

7. Koelmans, A. A. et al. Microplastics in freshwaters and drinking water: Critical review and assessment of data quality. Water Research 155, 410422 (2019).

8. Mammo, F. K., Amoah, I. D., Gani, K. M., Pillay, L., Ratha, S. K., Bux, F., \& Kumari, S. Microplastics in the environment: Interactions with microbes and chemical contaminants. Sci. Total Environ. 743, 140518 (2020).

9. Europe., P. Plastics - the Facts 2020. Plastics - the Facts 2020 (2020). Available at: https://www.plasticseurope.org/en/resources/publications/4312-plastics-facts-2020. (Accessed: 7th June 2021)

10. Shruti, V. C., Pérez-Guevara, F., Elizalde-Martínez, I., \& Kutralam-Muniasamy, G. First study of its kind on the microplastic contamination of soft drinks, cold tea and energy drinks - Future research and environmental considerations. Sci. Total Environ. 726, (2020).

11. Schwabl, P. et al. Detection of various microplastics in human stool: A prospective case series. Ann. Intern. Med. 171, 453-457 (2019).

12. Stock, V. et al. Impact of artificial digestion on the sizes and shapes of microplastic particles. Food Chem. Toxicol. 135, 111010 (2020).

13. Fackelmann, G. \& Sommer, S. Microplastics and the gut microbiome: How chronically exposed species may suffer from gut dysbiosis. Marine Pollution Bulletin 143, 193-203 (2019).

14. Jin, Y., Lu, L., Tu, W., Luo, T. \& Fu, Z. Impacts of polystyrene microplastic on the gut barrier, microbiota and metabolism of mice. Sci. Total Environ. 649, 308-317 (2019).

15. Lu, L., Wan, Z., Luo, T., Fu, Z. \& Jin, Y. Polystyrene microplastics induce gut microbiota dysbiosis and hepatic lipid metabolism disorder in mice. Sci. Total Environ. 631-632, 449-458 (2018).

16. Li, B. et al. Polyethylene microplastics affect the distribution of gut microbiota and inflammation development in mice. Chemosphere 244 , 125492 (2020).

17. Dupont, D. et al. Can dynamic in vitro digestion systems mimic the physiological reality? Critical Reviews in Food Science and Nutrition 59, 15461562 (2019).

18. Mackie, A., Mulet-Cabero, A. I. \& Torcello-Gomez, A. Simulating human digestion: Developing our knowledge to create healthier and more sustainable foods. Food Funct. 11, 9397-9431 (2020).

19. Cueva, C. et al. Application of a new Dynamic Gastrointestinal Simulator (SIMGI) to study the impact of red wine in colonic metabolism. Food Res. Int. 72, 149-159 (2015).

20. Miralles, B., del Barrio, R., Cueva, C., Recio, I. \& Amigo, L. Dynamic gastric digestion of a commercial whey protein concentratet. J. Sci. Food Agric. 98, 1873-1879 (2018).

21. Gil-Sánchez, I. et al. Application of the dynamic gastrointestinal simulator (simgi®) to assess the impact of probiotic supplementation in the metabolism of grape polyphenols. Food Res. Int. 129, 108790 (2020).

22. Gil-Sánchez, I. et al. Dynamic gastrointestinal digestion of grape pomace extracts: Bioaccessible phenolic metabolites and impact on human gut microbiota. J. Food Compos. Anal. 68, 41-52 (2018).

23. Tamargo, A., Cueva, C., Laguna, L., Moreno-Arribas, M. V. \& Muñoz, L. A. Understanding the impact of chia seed mucilage on human gut microbiota by using the dynamic gastrointestinal model simgi®. J. Funct. Foods 50, 104-111 (2018).

24. Tamargo, A., Martin, D., Navarro del Hierro, J., Moreno-Arribas, M. V. \& Muñoz, L. A. Intake of soluble fibre from chia seed reduces bioaccessibility of lipids, cholesterol and glucose in the dynamic gastrointestinal model simgi®. Food Res. Int. 137, 109364 (2020).

25. Barroso, E., Cueva, C., Peláez, C., Martínez-Cuesta, M. C. \& Requena, T. Development of human colonic microbiota in the computer-controlled dynamic SIMulator of the Gastrolntestinal tract SIMGI. LWT - Food Sci. Technol. 61, 283-289 (2015).

26. Brouwers, J. et al. Food-dependent disintegration of immediate release fosamprenavir tablets: In vitro evaluation using magnetic resonance imaging and a dynamic gastrointestinal system. Eur. J. Pharm. Biopharm. 77, 313-319 (2011).

27. Yin, N. et al. Investigation of bioaccessibility of $\mathrm{Cu}, \mathrm{Fe}, \mathrm{Mn}$, and $\mathrm{Zn}$ in market vegetables in the colon using PBET combined with SHIME. Sci. Rep. 7, 1-7 (2017).

28. Sun, G.-X., Van de Wiele, T., Alava, P., Tack, F. M. G. \& Du Laing, G. Bioaccessibility of selenium from cooked rice as determined in a simulator of the human intestinal tract (SHIME). J. Sci. Food Agric. 97, 3540-3545 (2017).

29. Cueva, C. et al. Gastrointestinal digestion of food-use silver nanoparticles in the dynamic SIMulator of the Gastrolntestinal tract (simgi®). Impact on human gut microbiota. Food Chem. Toxicol. 132, (2019). 
30. Lefebvre, D. E. et al. Utility of models of the gastrointestinal tract for assessment of the digestion and absorption of engineered nanomaterials released from food matrices. Nanotoxicology 9, 523-542 (2015).

31. Bahl, S. K., Cornell, D. D., Boerio, F. J. \& McGraw, G. E. Interpretation of the vibrational spectra of poly(ethylene terephtalate). J Polym Sci Part B Polym Lett 12, 13-19 (1974).

32. Štokr, J., Schneider, B., Doskočilová, D., Lövy, J. \& Sedláček, P. Conformational structure of poly(ethylene terephthalate). Infra-red, Raman and n.m.r. spectra. Polymer (Guildf). 23, 714-721 (1982).

33. Lin, C. C., Krommenhoek, P. J., Watson, S. S. \& Gu, X. Depth profiling of degradation of multilayer photovoltaic backsheets after accelerated laboratory weathering: Cross-sectional Raman imaging. Sol. Energy Mater. Sol. Cells 144, 289-299 (2016).

34. Adar, F. \& Noether, H. Raman microprobe spectra of spin-oriented and drawn filaments of poly(ethylene terephthalate). Polymer (Guildf). 26, 1935-1943 (1985).

35. Bistričić, L. et al. Raman spectra, thermal and mechanical properties of poly(ethylene terephthalate) carbon-based nanocomposite films. J. Polym. Res. 22, (2015).

36. Huerta Lwanga, E. et al. Decay of low-density polyethylene by bacteria extracted from earthworm's guts: A potential for soil restoration. Sci. Total Environ. 624, 753-757 (2018).

37. Lu, L. et al. Interaction between microplastics and microorganism as well as gut microbiota: A consideration on environmental animal and human health. Science of the Total Environment 667, 94-100 (2019).

38. Zhu, D. et al. Exposure of soil collembolans to microplastics perturbs their gut microbiota and alters their isotopic composition. Soil Biol. Biochem. 116, 302-310 (2018).

39. Yan, W., Hamid, N., Deng, S., Jia, P. P. \& Pei, D. S. Individual and combined toxicogenetic effects of microplastics and heavy metals (Cd, Pb, and Zn) perturb gut microbiota homeostasis and gonadal development in marine medaka (Oryzias melastigma). J. Hazard. Mater. $\mathbf{3 9 7}, 122795$ (2020).

40. Huang, J. N. et al. Exposure to microplastics impairs digestive performance, stimulates immune response and induces microbiota dysbiosis in the gut of juvenile guppy (Poecilia reticulata). Sci. Total Environ. 733, 138929 (2020).

41. Jin, Y. et al. Polystyrene microplastics induce microbiota dysbiosis and inflammation in the gut of adult zebrafish. Environ. Pollut. 235, 322-329 (2018).

42. Qiao, R. et al. Accumulation of different shapes of microplastics initiates intestinal injury and gut microbiota dysbiosis in the gut of zebrafish. Chemosphere 236, 124334 (2019).

43. Tuson, H. H. \& Weibel, D. B. Bacteria-surface interactions. Soft Matter 9, 4368-4380 (2013).

44. Yuan, J. et al. Microbial degradation and other environmental aspects of microplastics/plastics. Sci. Total Environ. 715, 136968 (2020).

45. Wang, K. et al. Gut microbiota protects honey bees (Apis mellifera L.) against polystyrene microplastics exposure risks. J. Hazard. Mater. 402, 123828 (2021).

46. Joo, S. et al. Structural insight into molecular mechanism of poly(ethylene terephthalate) degradation. Nat. Commun. 9, 1-12 (2018).

47. Lucas, N. et al. Polymer biodegradation: Mechanisms and estimation techniques - A review. Chemosphere 73, 429-442 (2008).

48. Yoshida, S. et al. A bacterium that degrades and assimilates poly(ethylene terephthalate). Science (80- ). 351, 1196-1199 (2016).

49. Finegold, S. M. Intestinal bacteria. The role they play in normal physiology, pathologic physiology, and infection. Calif. Med. 110, 455-9 (1969).

50. Luo, T. et al. Maternal Polystyrene Microplastic Exposure during Gestation and Lactation Altered Metabolic Homeostasis in the Dams and Their F1 and F2 Offspring. Environ. Sci. Technol. 53, 10978-10992 (2019).

51. Luo, T. et al. Maternal exposure to different sizes of polystyrene microplastics during gestation causes metabolic disorders in their offspring. Environ. Pollut. 255, (2019).

52. Hiippala, K. et al. Isolation of Anti-Inflammatory and Epithelium Reinforcing Bacteroides and Parabacteroides Spp. from A Healthy Fecal Donor. Nutrients 12, 935 (2020).

53. Nakano, V., Ignacio, A., Fernandes, M. R., Fukugaiti, M. H. \& Avila-Campos, M. J. Intestinal Bacteroides and Parabacteroides species producing antagonistic substances. (2006).

54. Gurung, M. et al. Role of gut microbiota in type 2 diabetes pathophysiology. EBioMedicine 51, 102590 (2020).

55. Magne, F. et al. The firmicutes/bacteroidetes ratio: A relevant marker of gut dysbiosis in obese patients? Nutrients 12, 1474 (2020).

56. Karlsson, F. H. et al. Gut metagenome in European women with normal, impaired and diabetic glucose control. Nature 498, 99-103 (2013).

57. Liang, D., Leung, R. K. K., Guan, W. \& Au, W. W. Involvement of gut microbiome in human health and disease: Brief overview, knowledge gaps and research opportunities. Gut Pathogens 10, 3 (2018).

58. D Mariat, O. F. F. L. V. G. H. S. J. D. G. C. J. F. The Firmicutes/Bacteroidetes ratio of the human microbiota changes with age. BMC Microbio/9, 123 (2009).

59. Shin, N.-R. R., Whon, T. W. \& Bae, J.-W. W. Proteobacteria: microbial signature of dysbiosis in gut microbiota. Trends Biotechnol. 33, 496-503 (2015). 
60. Kamada, N., Seo, S. U., Chen, G. Y. \& Núñez, G. Role of the gut microbiota in immunity and inflammatory disease. Nature Reviews Immunology 13, $321-335$ (2013).

61. Zhu, Q. et al. Dysbiosis signatures of gut microbiota in coronary artery disease. Physiol. Genomics 50, 893-903 (2018).

62. Wang, T. et al. Structural segregation of gut microbiota between colorectal cancer patients and healthy volunteers. ISME J. 6, 320-329 (2012).

63. Maldonado-Arriaga, B. et al. Gut Dysbiosis Is Related With Activity And Remission Phases Of Ulcerative Colitis And Healthy Condition. Res. Sq. (2020). doi:10.21203/rs.3.rs-28510/v1

64. Dahmus, J. D., Kotler, D. L., Kastenberg, D. M. \& Kistler, C. A. The gut microbiome and colorectal cancer: A review of bacterial pathogenesis. Journal of Gastrointestinal Oncology 9, 769-777 (2018).

65. Sheng, Q. et al. Characteristics of fecal gut microbiota in patients with colorectal cancer at different stages and different sites. Oncol. Lett. 18, 4834-4844 (2019).

66. Petrov, V. A. et al. Diagnostic potential of gut microbiota in Parkinson's disease. Bull. Sib. Med. 18, 92-101 (2019).

67. Das, T. et al. Alterations in the gut bacterial microbiome in people with type 2 diabetes mellitus and diabetic retinopathy. Sci. Rep. 11, 2738 (2021).

68. Li, Y. et al. The Gut Microbiota and Its Relevance to Peripheral Lymphocyte Subpopulations and Cytokines in Patients with Rheumatoid Arthritis. J. Immunol. Res. 2021, (2021).

69. Yong, C. Q. Y., Valiyaveetill, S. \& Tang, B. L. Toxicity of microplastics and nanoplastics in Mammalian systems. International Journal of Environmental Research and Public Health 17, 1509 (2020).

70. Ross, P. S. et al. Pervasive distribution of polyester fibres in the Arctic Ocean is driven by Atlantic inputs. Nat. Commun. 12, 1-9 (2021).

71. The European Commission. COMMISSION REGULATION (EU) N $N^{\circ} 10 / 2011$ of 14 January 2011 on plastic materials and articles intended to come into contact with food. Off. J. Eur. Union No 10/2011, (2011).

72. Fournier, E. et al. Microplastics in the human digestive environment: a focus on the potential and challenges facing in vitro gut model development. J. Hazard. Mater. 415, 125632 (2021).

73. Brodkorb, A. et al. INFOGEST static in vitro simulation of gastrointestinal food digestion. Nat. Protoc. 14, 991-1014 (2019).

74. Tamargo, A. et al. Influence of viscosity on the growth of human gut microbiota. Food Hydrocoll. 77, 163-167 (2018).

75. Callahan, B. J., Sankaran, K., Fukuyama, J. A., McMurdie, P. J. \& Holmes, S. P. Bioconductor Workflow for Microbiome Data Analysis: from raw reads to community analyses. F1000Research 5, 1492 (2016).

76. Callahan, B. J. et al. DADA2: High-resolution sample inference from Illumina amplicon data. Nat. Methods 13, 581-583 (2016).

77. Quast, C. et al. The SILVA ribosomal RNA gene database project: improved data processing and web-based tools. Nucleic Acids Res. 41, D590-6 (2013).

\section{Figures}




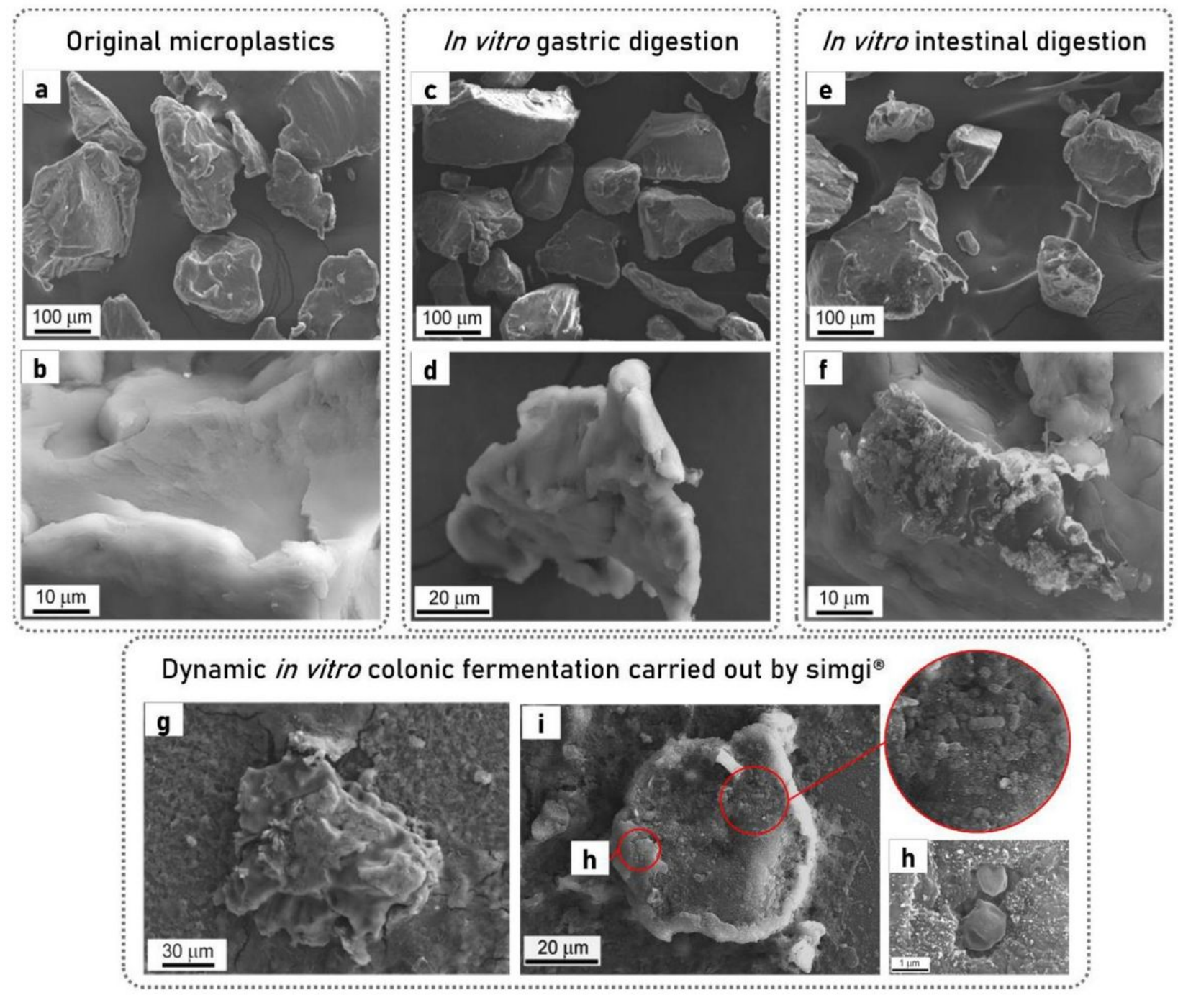

Figure 1

Micrographs by field emission scanning electron microscope (FESEM) of polyethylene terephthalate microplastics: a) and b) original PET microplastics (MPs), c) and d) PET MPs after in vitro gastric digestion, e) and f) PET MPs after in vitro gastrointestinal digestion, g), h) and i) PET MPs after in vitro gastrointestinal digestion and colonic fermentation. 

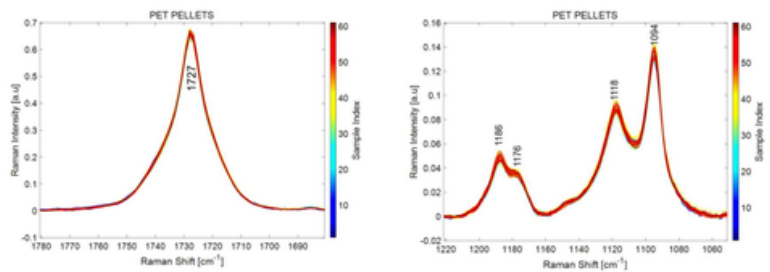

b)
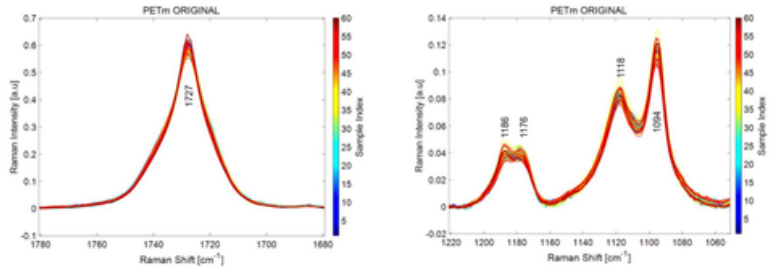

c)
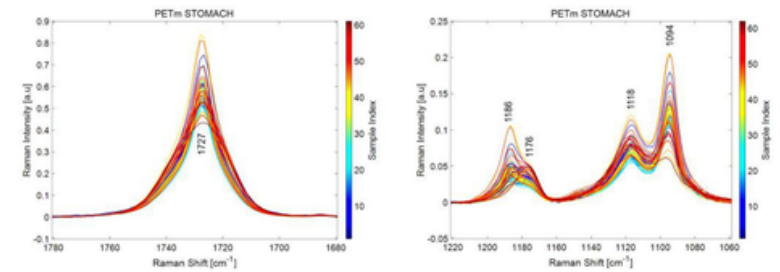

d) d)

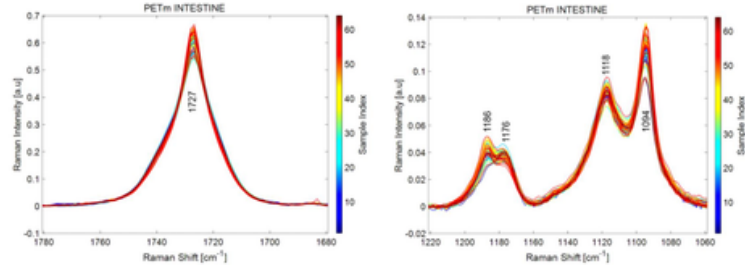

e)
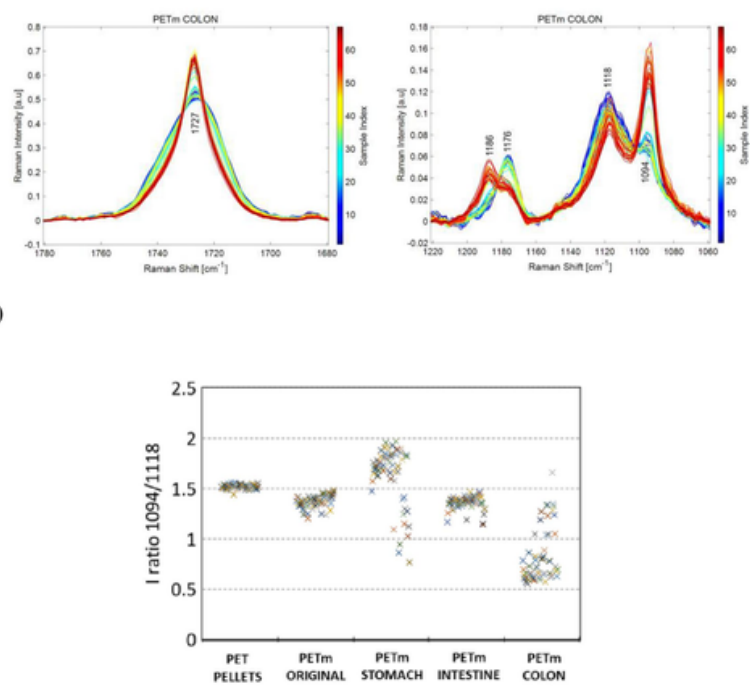

Figure 2

Raman spectra at 60-70 representative points of PET pellets a); original PET microplastics (MPs) b); PET MPs after in vitro gastric digestion c); PET MPs after in vitro gastrointestinal digestion d); and PET MPs after in vitro gastrointestinal digestion and colonic fermentation e). Figure 2.f) show the evolution of relative intensity of 1118 modes vs. $1094 \mathrm{~cm}-1$ during digestion simulation
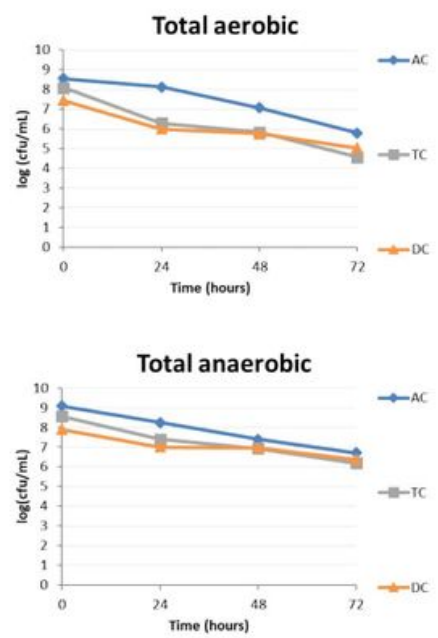
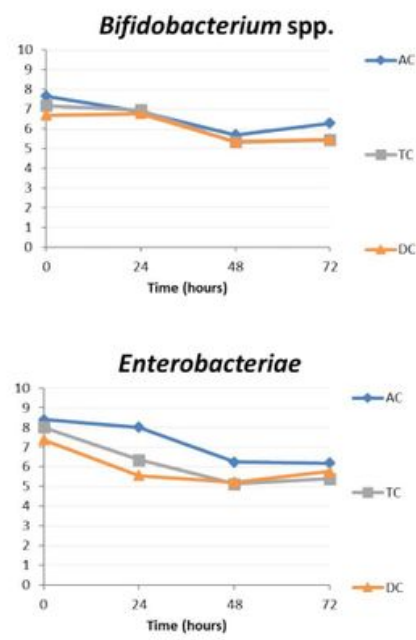

Lactic acid bacteria

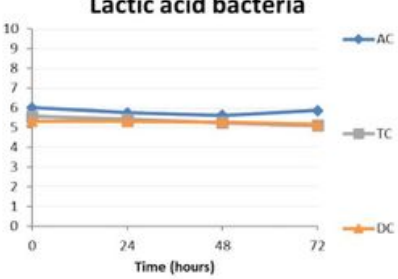

Staphylococcus spp.

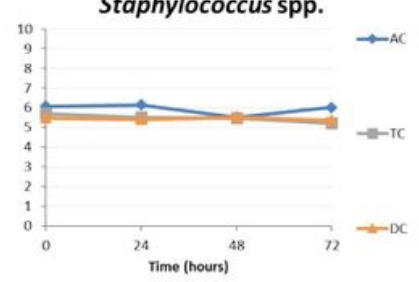

Clostridium spp.

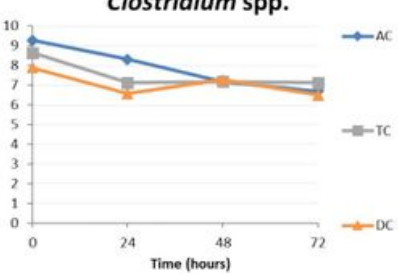

Enterococcus spp.

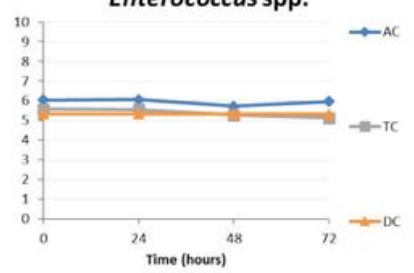

Figure 3

Evolution of the main microbial groups evaluated by plate counting for simgi® colonic compartments during colonic fermentation. AC: ascending colon; TC: transverse colon; DC: descending colon. CFU: Colony formatting units. 

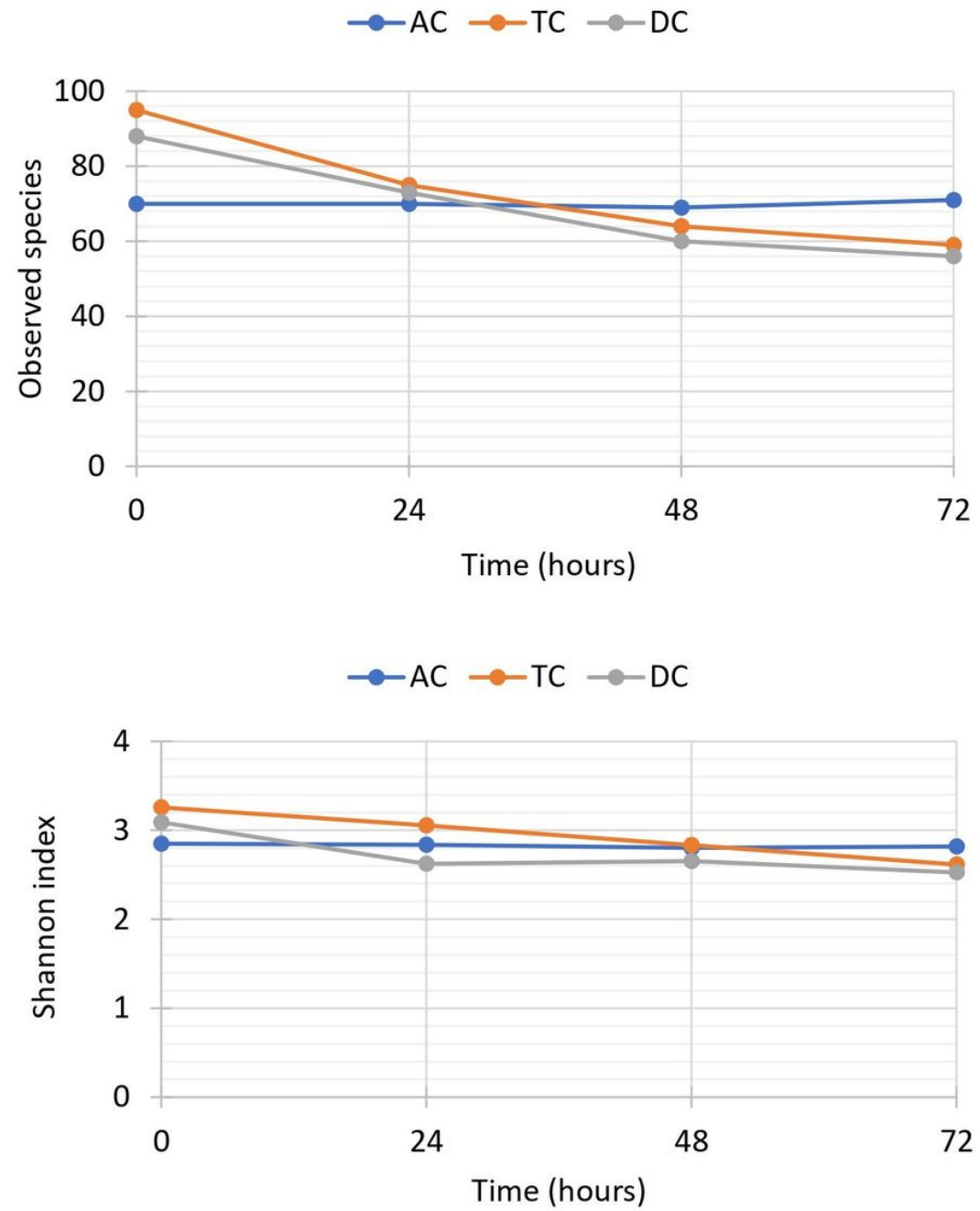

Figure 4

Alpha-diversity analysis for simgi ${ }^{\circledR}$ compartments during colonic fermentation. AC: ascending colon; TC: transverse colon; DC: descending colon. 


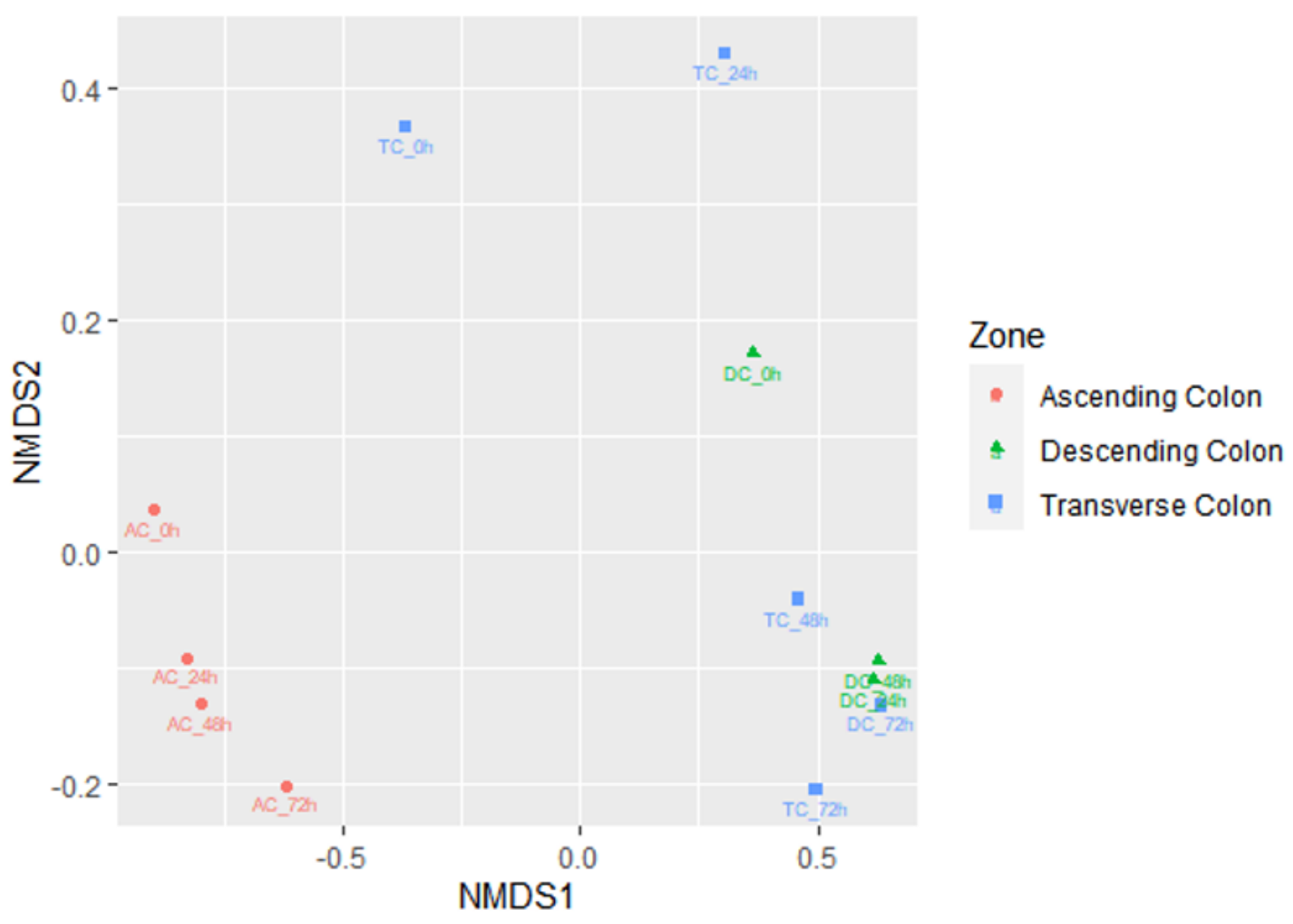

\section{Figure 5}

Beta-diversity analysis by non-metric multidimensional scaling (NMDS) for simgi® colonic compartments at different times (h) during fermentation. AC: ascending colon; TC: transverse colon; DC: descending colon.
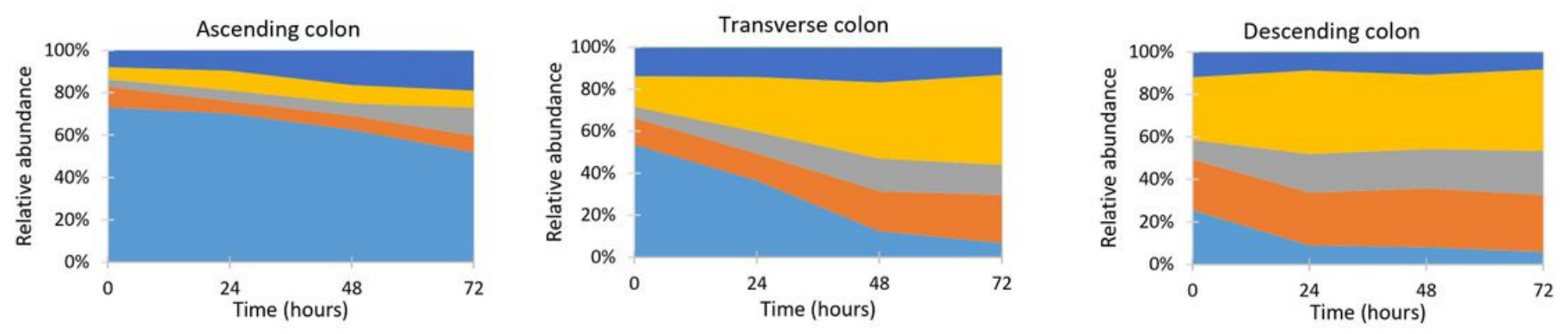

\# Bacteroidetes $=$ Proteobacteria $=$ Desulfobacteria

in Synergistetes $\quad$ Firmicutes $\quad$ Others

\section{Figure 6}

Evolution of the relative abundance at phylum level for the different simgi® compartments during colonic fermentation. Graphs show the taxa with a relative abundance $>0.5 \%$. 


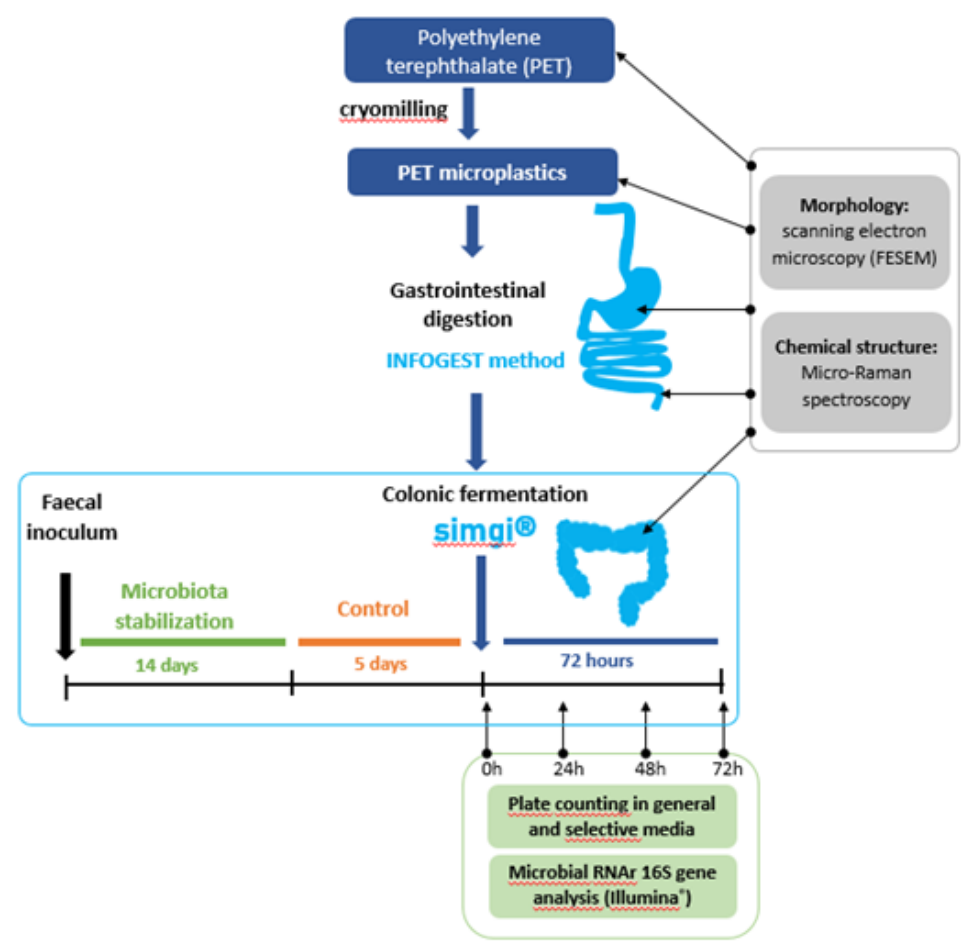

Figure 7

Experimental set-up of the study 